\title{
Costruire scenari per il futuro
}

\author{
Silvano Tagliagambe \\ (Professore Emerito di Filosofia della scienza, Universit di Sassari, \\ sil.tagliagambe@gmail.com)
}

\section{Una significativa contrapposizione}

Se si osserva con la dovuta attenzione ciò che si sta muovendo all'interno del panorama della ricerca scientifica contemporanea non si può fare a meno di rilevare una significativa contrapposizione tra ciò che emerge dai risultati delle neuroscienze e alcune tendenze che si riscontrano in seguito alla prepotente irruzione sulla scena delle complesse questioni legate ai big data e agli approcci richiesti da una loro efficace utilizzazione. Questa antitesi incide in modo significativo sul modo di immaginare il mondo di domani e sulla scelta degli strumenti concettuali più idonei per costruirlo con efficacia: ed è per questo che occorre farne oggetto di specifica attenzione e di un'analisi approfondita.

1.1. La percezione come selezione e il progetto Le ricerche sui processi cerebrali, e in particolare i risultati conseguiti dal gruppo dell'università di Parma guidato da Giacomo Rizzolatti, culminati nella scoperta dei neuroni mirror, stanno erodendo sempre più la credibilità del classico schema percezione $\Rightarrow$ cognizione $\Rightarrow$ movimento, che è messa irrimediabilmente in crisi dalla constatazione del fatto che la percezione appare immersa nella dinamica dell'azione, risultando, di conseguenza, ben più articolata e composita di come in passato è stata presentata. A questa conclusione si è pervenuti grazie a ricerche che hanno permesso di evidenziare come il sistema motorio non sia affatto periferico e isolato dal resto delle attività cerebrali, bensì consista di una complessa trama di aree differenziate per localizzazione e funzioni, e in grado di fornire un apporto decisivo a realizzare quelle trasformazioni sensorimotorie da cui dipendono l'individuazione, la localizzazione degli oggetti e l'attuazione dei movimenti richiesti dalla maggior parte degli atti e dei comportamenti nei quali si articola la nostra esperienza quotidiana. Gli aspetti interessanti che emergono già oggi da questi risultati sono molteplici. Il primo è che esiste una capacità, basata su precisi meccanismi neuronali, di "tradurre" in modo immediato la prospettiva corporea di chi esegue una determinata azione in quella di chi la osserva. Questo significa che per operare questa traduzione non abbiamo affatto bisogno della mediazione di un "dizionario", costituito dalle rappresentazioni mentali, così come 
vorrebbe il cognitivismo classico, che concepisce la mente come un sistema funzionale, i cui processi possono essere descritti come manipolazioni di simboli informazionali, sulla base di una serie di regole sintattiche formali. Se le cose stessero veramente così ne scaturirebbe l'idea che le rappresentazioni siano intrinsecamente simboliche e che il pensiero debba essere ridotto a un processo meramente computazionale. ${ }^{1}$

Per chiarire fino in fondo la differenza tra l'impostazione del cognitivismo e quella che viene accreditata dalle scoperte dell'équipe di Rizzolatti può essere utile fare un esempio. Se in un bar vedo qualcuno dirigere la mano verso un boccale di birra, comprenderò immediatamente che egli sta per sorseggiare quella bevanda. Il punto cruciale è: come faccio? Secondo l'approccio cognitivista classico per arrivare a questa conclusione debbo necessariamente tradurre le informazioni sensoriali relative al gesto della persona che osservo in una serie di rappresentazioni mentali, che condividono col linguaggio lo stesso formato preposizionale: nel caso specifico, queste rappresentazioni riguarderanno il desiderio dell'altro di bere birra, le sue credenze circa il fatto che il boccale che sta per afferrare sia effettivamente pieno di questa bevanda, e la sua intenzione di portare il bicchiere alla bocca per bere. La scoperta dei neuroni specchio ci consente invece di dire che l'osservazione di un'azione induce l'attivazione dello stesso circuito nervoso deputato a controllarne l'esecuzione: l'osservazione dell'azione induce quindi nell'osservatore l'automatica simulazione della stessa azione e, attraverso quest'ultima, la sua comprensione.

Questo schema esplicativo è denso di conseguenze importanti. La prima è che è impossibile la conoscenza di altre persone e di ciò che fanno indipendentemente da noi stessi e, in particolare, dal riferimento al nostro corpo, ai suoi movimenti. La "realtà dell'altro" non può essere conosciuta in quanto tale, ma solo in stretta relazione al soggetto che la percepisce e la osserva e che interagisce con essa. Interessante, da questo punto di vista, è l'ipotesi avanzata da Gallese, uno dei componenti dell'équipe di Parma cui si deve la scoperta dei neuroni mirror, il quale predice "l'esistenza di neuroni mirror 'somato-sensoriali' che potrebbero contribuire a darci la capacità di identificare le diverse parti corporee altrui, riferendole a parti equivalenti del nostro corpo" (Gallese 2003, 39).

Questa ipotesi è stata effettivamente corroborata dai risultati degli esperimenti effettuati dallo stesso Gallese in collaborazione con altri colleghi (Keysers, Wickers, Gazzola, Anton, Fogassi, Gallese 2004), i quali mostrano che l'esperienza di essere toccati in una parte del proprio corpo determina l'attivazione dello stesso circuito neurale attivato dall'osservazione del corpo di qualcun altro che viene toccato in una parte corporea equivalente, la regione corticale implicata è l'area SII/PV, localizzata nell'opercolo parietale,

${ }^{1}$ Per una critica al cognitivismo si veda Tagliagambe (2002, in particolare il cap. 5, 93105). 
comunemente ritenuta un'area esclusivamente tattile. La medesima regione corticale viene quindi attivata sia quando esperiamo in prima persona una sensazione tattile localizzata ad una parte del nostro corpo, che quando siamo testimoni di un'analoga esperienza sensoriale esperita da qualcun altro.

La seconda delle conseguenze di questo approccio è il mutamento rilevante dell'idea di percezione che ne scaturisce. Il significato di questo cambiamento di scenario appare chiaro se ci riferiamo a un'importante nozione introdotta nel 1979 da James J. Gibson, quella di affordance, o risorsa, coniata per descrivere il rapporto reciproco tra un animale e l'ambiente, e divenuta successivamente uno dei cardini della psicologia ecologica. Secondo Gibson la percezione visiva di un oggetto comporta l'immediata e automatica selezione delle proprietà intrinseche che ci consentono, di volta in volta, di interagire con esso. Queste "non sono delle proprietà fisiche astratte, ma incarnano delle opportunità pratiche che l'oggetto, per così dire, offre all'organismo che lo percepisce" (Gibson 1979 [1999], 206).

Per esempio, uno scultore può percepire una pietra interpretandola come una figura in essa nascosta; un muratore come elemento da sovrapporre ad altri elementi simili, per innalzare un muro; un bambino come un oggetto da gioco, da far rotolare per la strada; un collezionista di pietre come un campione pregevole da affiancare su una mensola ad altri campioni di diversa forma e diversamente colorati.

La stessa cosa avviene con gli artefatti. Possiamo percepire un martello come qualcosa da usare per piantare un chiodo nella parete, ma anche come una leva per svellere un pezzo di legno incollato su un ripiano; o come un livellatore, per appiattire un materiale morbido, ma difficilmente modellabile, come una lastrina di stagno, su una superficie più dura; come un'arma per colpire qualcuno che ci ha aggredito; oppure uno strumento adatto a rompere un salvadanaio o per ripulire la suola di una scarpa da qualcosa che vi si è appiccicato. Insomma la sua funzione e il suo significato possono variare a seconda delle esigenze di chi lo maneggia e che lo può quindi vedere in molti modi tra loro alternativi.

Tutti gli oggetti, anche i più semplici e banali, contengono pertanto più di un'affordance. Nel caso di una comune tazzina le affordances visive offerte al nostro sistema motorio riguardano il manico, il corpo centrale, il bordo superiore, ecc. Di conseguenza, l'osservazione di essa:

determinerà l'attivazione di più popolazioni neurali nell'area intraparietale superiore (AIP), ciascuna delle quali codifica una determinata affordance. È verosimile che queste 'proposte' di azione possano essere inviate all'area F5, innescando veri e propri atti motori potenziali. Ora la scelta di come agire non dipenderà soltanto dalle proprietà intrinseche dell'oggetto in questione (forma, taglia, orientamento), bensì anche da quello che intendiamo fare di esso, dalle funzioni d'uso che gli riconosciamo, ecc. Nel caso della tazzina, per esempio, la 
afferreremo in modi differenti se vogliamo prenderla per bere un caffè, per sciacquarla $\mathrm{o}$, più modestamente, per spostarla. E già nel primo caso la presa potrà essere diversa a seconda che temiamo di scottarci o meno, degli eventuali oggetti che circondano la tazzina, delle nostre abitudini, della nostra inclinazione a rispettare le buone maniere, ecc. (Rizzolatti \& Sinigaglia 2006, 36).

Possiamo pertanto dire che ci troviamo di fronte a una coppia di tendenze $e$ capacità, entrambe effettuali, cioè presenti e attive nello spazio e nel tempo. La tazzina del nostro esempio mette a disposizione di chi la vuole utilizzare, come risorse proprie, tutta una serie di possibilità di presa le quali esistono oggettivamente, sia che vengano percepite o no, e che appaiono caratterizzate, appunto, da tendenze oggettive; d'altro canto esiste una capacità soggettiva, ma altrettanto reale ed effettiva, da parte dell'uomo, di estrapolare ed elaborare le informazioni relative alla forma, alla taglia e all'orientamento del manico, del bordo superiore, ecc., che rientrano nel processo di selezione, da parte sua, delle modalità di presa, e di attivare la serie di movimenti (a cominciare da quelli relativi alla prefigurazione della mano) che di volta in volta intervengono nell'atto di afferrarla. Dall'accoppiamento di queste due serie di tendenze oggettive emerge come "la tazzina funga [...] da polo d'atto virtuale, che per la sua natura relazionale definisce ed è insieme definito dal pattern motorio che viene ad attivare" (Rizzolatti \& Sinigaglia 2006, 47).

Dall'altra parte, cioè da quella dell'uomo che si pone di fronte alla tazzina, si ha un vedere che non è fine a sé stesso, ma è orientato a guidare la mano, e che si presenta, dunque, "anche, se non soprattutto, un vedere con la mano, rispetto al quale l'oggetto percepito appare immediatamente codificato come un insieme determinato di ipotesi d'azione" (Rizzolatti \& Sinigaglia 2006, 49). La percezione, dunque, si presenta come un'implicita preparazione dell'organismo a rispondere e ad agire e dalla quale scaturisce, di conseguenza, un tipo di comprensione che ha una natura eminentemente pragmatica, che non determina di per sé alcuna rappresentazione "semantica" dell'oggetto, in base alla quale esso verrebbe, per esempio, identificato e riconosciuto come una tazzina da caffè, e non semplicemente come qualcosa di afferrabile con la mano).

Il fatto che le due serie di tendenze effettuali sulle quali ci siamo soffermati assumano significato e valore solo nella loro reciproca interazione dà un senso preciso e concreto all'idea che al pensiero oggettivante, fondato su una pretesa autonomia e autosufficienza delle "cose" che popolano il nostro ambiente, debba subentrare un'ontologia delle relazioni, in virtù della quale, appunto, la tazzina, più che un oggetto a sé stante, risulta essere, come si è visto, un polo d'atto virtuale al quale corrisponde uno spettro, altrettanto virtuale, di modalità di presa e di relativi movimenti. 
Questo spostamento da un'ontologia delle proprietà, basato sull'idea dell'esistenza di cose autonome e a sé stanti, dotate di specifici tratti distintivi, che successivamente entrano in rapporti reciproci e interagiscono, a un'ontologia delle relazioni, secondo la quale invece sono le relazioni che danno origine alla cose e non viceversa, per cui le cose cambiano solo in rapporto l'una all'altra e il mondo è una rete di correlazioni e di reciproche informazioni tra sistemi fisici, è importante perché ci consente di superare la coppia opposizionale reale/virtuale, a cui subentra la contrapposizione al virtuale e al possibile dell'effettuale ${ }^{2}$.

Per il passaggio dall'intera cerchia dei possibili (nel caso della tazzina da caffè dallo spettro complessivo delle affordances visive da essa offerte) all'effettualità dell'uso che viene concretamente attivato risulta decisiva, come detto, la relazione con il sistema motorio di chi intende utilizzare questo specifico oggetto. Dal quadro generale proposto dagli sviluppi delle neuroscienze emerge dunque l'incidenza primaria del gesto nel momento stesso in cui si dispiega e si attua, di quel "gesto che racconta" il quale svolge la funzione di ponte tra il sistema motorio, il linguaggio e il ragionamento, tra il corpo, le parole e i concetti. Ne scaturisce un sincronismo tra agire, pensare e parlare che confuta il modello tradizionale di un processo di elaborazione delle informazioni sensoriali in entrata che, sviluppandosi in modo lineare, si conclude con la produzione di un'uscita motoria, di un'azione. Quest'ultima, invece, non è l'esito finale e la meccanica dell'esecuzione del processo percettivo, ma è parte integrante di questo processo e inscindibile dallo stimolo sensoriale, in quanto contenuta in esso. Su questi risultati si fonda una fisiologia dell'azione che conferisce inedita dignità teorica alle operazioni concrete, alla manipolazione, al "pensare con le mani".

A supporto di questa conclusione c'è la scoperta del fatto che ogni azione, qualunque essa sia, è caratterizzata dalla presenza di uno scopo. Gli stessi movimenti, come flettere le dita di una mano, possono essere eseguiti per

${ }^{2}$ Questo spostamento fa ovviamente riferimento a uno degli apporti più significativi della rivoluzione copernicana di Kant, vale a dire la differenza, che viene tracciata nella Critica della ragion pura, tra la Realität, categoria della qualità, corrispondente al giudizio affermativo, da una parte, e il concetto di Dasein e quelli di Existenz e di Wirklichkeit, cioè di esistenza e di effettualità, strettamente associati a esso, dall'altra, che rientrano invece nell'ambito delle categorie della modalità. Ciò che emerge da questa distinzione è che la realtà in quanto categoria della qualità non si riferisce all'esistenza effettiva di un qualcosa nel mondo esterno, bensì alle determinazioni e ai contenuti che sono propri di un qualcosa in quanto res, cioè alla determinazione del contenuto di una cosa in quanto cosa. Come sottolinea Heidegger, quando ci riferiamo alla realtà così intesa e definita "noi guardiamo alla cerchia dei possibili aspetti come tale, e, più esattamente, a ciò che traccia i limiti di questa cerchia, a ciò che regola $\mathrm{e}$ delinea il modo in cui qualcosa deve apparire in generale, per poter offrire la veduta corrispondente" (Heidegger 1962, 127). 
conseguire fini diversi (afferrare una tazzina, grattarsi il capo, giocherellare con le dita ecc.). La presenza di scopi diversi fa di quegli stessi movimenti degli atti motori diversi.

Il sistema motorio non è pertanto un semplice controllore di movimenti: alla base della sua organizzazione funzionale c'è la nozione teleologica di scopo. Ciò che fa di un movimento un'azione è il finalismo, vale a dire il progetto d'azione e lo scopo, che sono alla base del modo con cui il nostro sistema cervellocorpo-mente struttura e organizza la nostra interazione con il mondo.

La correlazione di azione e scopo emerge ancora più chiaramente da una serie di esperimenti in cui gli stessi neuroni premotori dell'area F5 sono stati registrati mentre la scimmia afferrava oggetti con una pinza che, per la sua particolare conformazione, la obbligava a eseguire movimenti della mano opposti a quelli normalmente impiegati per afferrare un pezzo di cibo; i neuroni per l'afferramento continuavano a scaricare durante l'afferramento del cibo con la pinza, anche se il conseguimento dello scopo era raggiunto impiegando movimenti del tutto opposti a quelli naturali. Si tratta di acquisizioni che corroborano la conclusione che ciò che tali neuroni rappresentano/controllano è lo scopo dell'atto motorio e non i mezzi, cioè i movimenti, richiesti per conseguirlo. In questo modo le immagini del corpo che reagisce agli stimoli e all'incidenza dell'ambiente, modificandosi, si integrano con quelle che sono invece il risultato dell'azione proattiva del soggetto, delle sue decisioni e scelte degli oggetti con i quali interagire e del modo di farlo, in base a un preciso principio di "coerenza" col proprio sentire nel contesto determinato e nella specifica situazione in cui si trova.

Questi risultati pongono, pertanto, il progetto d'azione e lo scopo che s'intende perseguire tramite esso all'inizio, e non a conclusione del processo percettivo $e$ dell'entrata in scena delle funzioni cognitive superiori da esso innescato. È infatti sulla base di questo progetto e delle sue finalità che si provvede a selezionare, tra tutte le risorse informative che l'ambiente ci mette a disposizione, quelle che appaiono funzionali al progetto medesimo e pertinenti rispetto a esso. Ancora una volta e a maggior ragione la percezione, più che risultato di un processo di generalizzazione induttiva che porta al riconoscimento delle forme e all'assegnazione di uno specifico oggetto all'insieme di cui fa parte ("Questa che ho di fronte è una bottiglia"), appare dunque il risultato di una selezione diretta a discriminare tra informazioni pertinenti e informazioni non pertinenti. Non può trattarsi, ovviamente, di una distinzione valida in assoluto, dal momento che non sussistono dati e informazioni che siano pertinenti o non pertinenti, significative o non significative, ridondanti o no, superflue o no in assoluto, ma sempre relativamente e rispetto al preciso scopo che s'intende perseguire e al progetto d'azione che si vuole attuare. Le immagini "proattive" del corpo che agisce si saldano con il progetto d'azione e con il movimento che viene compiuto per attuarlo e si nutrono così dell'ulteriore significato derivante da questa sua fusione col gesto, che a sua volta, in seguito a questa 
relazione con il linguaggio iconico, diventa, come si è detto, un gesto con una precisa valenza narrativa, un gesto che racconta.

È dunque la centralità del progetto d'azione e la sua funzione di selezione, tra tutte le informazioni disponibili, di quelle pertinenti rispetto a esso, a rendere ancora più chiaro il passaggio dalla coppia opposizionale reale/virtuale a quella effettuale/virtuale. Progettare significa infatti riuscire a vedere $e$ a pensare altrimenti l'effettualità (l'oggetto che si ha di fronte, qui e ora, nello spazio e nel tempo) cogliendo le alternative della sua modalità di presentazione, insite nel suo specifico orizzonte di realtà. Così facendo non si esce, ovviamente, dalla totalità della determinazione possibile dell'oggetto medesimo, cioè dalla sua realtà: si va invece al di là dello specifico modo in cui si è abituati a considerarlo sulla base delle modalità percettive usuali ed egemoni. Quelle che Leopardi, non a caso, invita a superare, integrandole con l'immaginazione:

All'uomo sensibile e immaginoso, che viva, come io sono vissuto gran tempo, sentendo di continuo ed immaginando, il mondo e gli oggetti sono in certo modo doppi. Egli vedrà cogli occhi una torre, una campagna; udrà cogli orecchi un suono d'una campana; e nel tempo stesso coll'immaginazione vedrà un'altra torre, un'altra campagna, udrà un altro suono. In questo secondo genere di obbietti sta tutto il bello e il piacevole delle cose. Trista quella vita (ed è pur tale la vita comunemente) che non vede, non ode, non sente se non che oggetti semplici, quelli soli di cui gli occhi, gli orecchi e gli altri sentimenti ricevono la sensazione (Leopardi, Zibaldone di pensieri, Firenze 30 novembre 1828).

\subsection{Big data e la fine della teoria}

A questa centralità del progetto e delle capacità immaginative e costruttive dell'uomo si contrappone una prospettiva all'origine della quale vi è il famoso post di Anderson del 2008 su Wired "The End of Theory: The data Deluges Makes the Scientific Method Obsolete". L'autore basa la sua tesi sull'assunto che la straordinaria potenza di calcolo oggi a disposizione, grazie alla quale siamo ormai in condizione di analizzare basi dati enormi a basso costo e in tempi sempre più rapidi, consente un apprendimento di tipo statistico che avviene in assenza di una reale comprensione dei fenomeni. Così funzionano gli algoritmi di deep learning e le reti neurali.

Sarebbe questa "la fine della teoria". I dati, secondo Anderson, parlano da soli, al punto che non abbiamo bisogno di alcuna chiave interpretativa per leggerli e di alcun modello per rappresentarli: essi sono autosufficienti, bastano per comprendere i fenomeni naturali e sociali. Del resto i modelli non sono che approssimazioni grossolane alla verità, caricature di una più complessa realtà sottostante, utili agli scienziati per visualizzare nella loro mente i fenomeni studiati ma tutt'altro che indispensabili. Sulla base di queste premesse si è arrivati a ipotizzare una ormai prossima rivoluzione scientifica, quella derivante dalla possibilità di fare scienza attraverso la sola analisi dei dati. Si 
tratterebbe di un quarto paradigma in ordine di tempo da aggiungersi a quelli che si sono fin qui succeduti: il metodo sperimentale, quello teorico matematico e quello computazionale, basato sulle simulazioni numeriche (Hey, Tansley \& Tolle 2009).

Questa posizione, per essere ben compresa, esige un primo chiarimento, riguardante la natura e la funzione dei modelli. Per molto tempo la fisica si è basata su un tacito postulato, secondo il quale la comprensione dei fenomeni esigerebbe la rappresentazione visiva, assicurata appunto da modelli capaci di fornirla. Questo tesi è stata contrastata con molta decisione e chiarezza da Dirac, il quale nella prefazione alla prima edizione (del 1930) della sua opera $I$ principi della meccanica quantistica osserva che

la tradizione classica era di considerare l'universo come un'associazione di enti osservabili (particelle, fluidi, campi, ecc.) in moto secondo definite leggi di forze, in modo da poterci formare, dell'intero schema, un modello mentale nello spazio e nel tempo. Ciò portò a una fisica il cui scopo era quello di fare delle ipotesi sul meccanismo e sulle forze che connettevano questi enti osservabili, in modo da rendere ragione del loro comportamento nella maniera più semplice possibile. Negli ultimi tempi, però, è divenuto sempre più evidente che la natura si comporta in maniera diversa. Le sue leggi fondamentali non governano in un modo molto diretto l'universo quale appare nel nostro modello mentale, ma controllano invece un substrato di cui non possiamo formarci un modello mentale senza introdurre inesattezze irrilevancies, che può forse essere meglio reso con "qualcosa di non pertinente"] (Dirac 1959, xi).

A giudizio di Dirac non c'è comunque nessun motivo di considerare insoddisfacente questa situazione, malgrado la distanza che la separa dall'ideale coltivato dalla tradizione e dal realismo classici. A proposito di questi ultimi si può infatti rilevare che:

il principale scopo della fisica non è di fornire modelli, bensì di formulare delle leggi che governino i fenomeni e la cui applicazioni porti alla scoperta di nuovi fenomeni. Se poi esiste un modello, tanto meglio; ma l'esistenza o no di esso è questione di secondaria importanza. Nel caso dei fenomeni atomici, infatti, non ci si deve aspettare che esista alcun 'modello' nel senso abituale della parola, cioè di qualcosa che funzioni essenzialmente su linee classiche. Si può tuttavia estendere il significato della parola 'modello' per includervi qualsiasi maniera di raffigurarsi le leggi fondamentali in modo da rendere ovvia la loro autocompatibilità [self-consistency] (Dirac 1959, 14; il corsivo è mio).

È chiaro che, con questa estensione, la parola "modello" viene a essere svincolata dal riferimento imprescindibile all'esigenza di un'immagine in senso stretto: la non-contraddittorietà delle leggi può benissimo essere 
evidenziata senza chiamare in causa il postulato dell'inevitabile riferimento a una "rappresentazione visiva". A giudizio di Dirac è pertanto necessario liberarsi da questo postulato e accettare il fatto che i dati sperimentali, negli ordini di grandezza molto grandi e molto piccoli, non danno necessariamente luogo a immagini, e noi dobbiamo imparare a farne a meno.

I fisici generalmente erano soliti usare il termine "modello" per riferirsi non già a una qualche forma di calco materiale della realtà, bensì a una sua riproduzione ideale e concettuale, tesa a rendere visibile l'invisibile, cioè a trasformare in entità visualizzabili strutture e organizzazioni non direttamente accessibili per un motivo qualunque, come le loro ridotte dimensioni, alla percezione diretta. È a questo scopo che venne proposto, ad esempio, il modello planetario dell'atomo, che quando fu creato e per alcuni anni successivi fu concepito come la riproduzione di una realtà troppo piccola per essere visibile, ma tale comunque da cogliere i tratti distintivi essenziali di quest'ultima, come avremmo potuto verificare se fossimo stati in condizione di ingrandirla.

Questa illusione di fedeltà era però destinata a durare poco: come sottolinea appunto Dirac è stato, in particolare, lo sviluppo della meccanica quantistica a costringere a prendere atto del fatto che le particelle microscopiche non hanno le stesse proprietà dei corpi macroscopici con i quali abbiamo familiarità e che, di conseguenza, il cambiamento di scala, con il passaggio dal livello della realtà delle "palline" visibili, localizzabili e aventi una certa forma ecc., a quello delle particelle subatomiche, comporta un mutamento di scenario tale che qualsiasi tentativo di assumere le prime per rappresentare e visualizzare le seconde vuol dire falsare la realtà.

Si rafforzò allora la convinzione, già emersa in precedenza, che al modello non andasse più associato il requisito della fedeltà, rispetto a ciò che veniva rappresentato per il suo tramite, e che dunque esso andasse considerato come un sistema fittizio visualizzabile, il cui vantaggio consisteva proprio nel rendere in qualche modo percepibile quell'invisibile semplice" che si sostituisce al "visibile complicato" e ci consente di spiegarlo.

Come osserva Toraldo di Francia:

Una volta persa la perfetta similitudine con la realtà, era però inevitabile che il modello subisse un'ulteriore evoluzione e diventasse puramente astratto. $E$ infatti nella fisica contemporanea delle particelle, un modello è quasi sempre un modello matematico, che non ha molto a che fare con la visualizzazione. Non è una vera e propria teoria perché si è lungi dall'aver dimostrato che va d'accordo con tutti i fatti sperimentali noti. E spesso non è nemmeno un'ipotesi, perché si sa bene che descrive al più un aspetto particolare della realtà, rimanendo scollegato e forse addirittura in contraddizione con le teorie precedenti, già acquisite. Si tratta molto spesso di uno strumento avente soprattutto valore euristico (Toraldo di Francia 1976, 8081). 
Questo valore euristico non è pertanto legato alla rappresentazione visiva, intesa nel senso usuale, di un fenomeno qualunque $X$, oggetto di studio, ma piuttosto alla costruzione di un'analogia tra questo fenomeno X (ad esempio un territorio) e un oggetto costruito $\mathrm{M}$ (la sua mappa) che permette, in quanto simula $\mathrm{X}$, di rispondere a un qualche quesito $\mathrm{P}$, posto a riguardo di quest'ultimo. Le legittimità del modello in questo caso non è legata né alla sua capacità di visualizzare alcunché, né alla sua veridicità, intesa come rappresentazione fedele ed esaustiva, bensì al rispetto delle seguenti condizioni:

1) che M abbia una sua coerenza interna;

2) che la costruzione di $M$ sia determinata dall'esigenza di trovare una risposta al problema $\mathrm{P}$ concernente $\mathrm{X}$;

3) che questo problema sia traducibile in un problema $P^{\prime}$, concernente $M$, il che significa possibilità di tenere costantemente sotto controllo l'analogia $\mathrm{X}$-M tra un fenomeno e un oggetto (teorico-formale) costruito con un certo linguaggio;

4) che la soluzione $S^{\prime}$ al problema $P^{\prime}$ che il modello ha consentito di escogitare possa, a sua volta, venire trasferita al problema originario $\mathrm{P}$, con conseguente individuazione di una soluzione $S$ applicabile a esso. Questo risultato, ovviamente, va poi sottoposto a una verifica sperimentale attraverso il meccanismo popperiano della corroborazione/falsificazione);

5) che il carattere esplicativo del modello, che si esprime proprio in questa sua capacità di trovare la soluzione cercata, si manifesti anche sotto forma di produzione di un livello più alto e astratto di "visualizzazione", nel senso che esso, facendo intervenire processi tra entità invisibili (la sostituzione al visibile complicato di una struttura o un meccanismo più semplice, non osservabile a livello di evidenza fenomenologica, che generalmente caratterizza la costruzione di M) permetta di ricostruire, a uno stadio più elevato, la morfologia visibile.

In queste condizioni possiamo trovare i sei criteri di giustificazione dei modelli usualmente invocati e proposti: coerenza razionale, rispondenza ai dati sperimentali, unicità, minimalità, falsificabilità, potere di previsione. Non solo, ma esse segnalano il distacco da ogni tentazione riduzionistica, in quanto legano l'esistenza stessa del modello e la sua legittimità alla possibilità, che si realizza grazie alla elaborazione di esso, di trovare una risposta a un problema riguardante l'oggetto di riferimento, a cui la validazione del modello rimanda dunque ineluttabilmente.

Riferita a questa seconda funzione dei modelli la posizione di Anderson esprime dunque la convinzione che il paradigma dei big data sia in grado di riportare il discorso scientifico sul piano dell'oggettività dei fenomeni osservati (lo studio diretto del territorio nella metafora proposta), senza bisogno di riferirsi ad alcuna mappa e all'orizzonte di attese dell'osservatore, in quanto non vi è alcun benchmark, parametro o punto di riferimento fissato a 
priori. Con i big data si lascia che siano i dati a dirci quali sono il benchmark, la correlazione, il modello. I dati ci svelano il loro segreto, a prescindere dall'ipotesi di partenza, che diventa pertanto irrilevante. Essi ci fanno dunque vedere come stanno i fatti, senza alcuna pregiudiziale. Le cose stanno così perché sono i dati a dircelo; sono loro a segnalarci le correlazioni significative fra un numero tendenzialmente infinito di variabili.

Questo punto di vista è stato di recente contestato da Dominique Cardon (2016), il quale mette sotto processo molti discorsi relativi ai big data, espressione ormai diventata d'uso comune per descrivere una raccolta di dati così estesa in termini di volume, velocità e varietà da richiedere tecnologie $\mathrm{e}$ metodi analitici specifici per l'estrazione del loro significato. In proposito egli sottolinea che di big data si può discutere a patto di mantenere le distanze da certe mitologie che riempiono i discorsi dei loro promotori. La madre di tutte queste mitologie è quella che vede, con l'avvento dei big data, la nascita di una nuova stagione, nella quale i dati sono finalmente liberi di parlarci nella loro oggettività. A giudizio dell'autore si tratta di un'illusione o, quel che è peggio, di un discorso in cattiva fede, in quanto i modelli statistici standard sono incapaci di rappresentare la realtà senza agire su di essa. Misurare significa fissare indicatori e categorie, ossia definire un orizzonte di attese. Ma ciò orienta il comportamento degli attori sociali sottoposti a misurazione. La società adatta inevitabilmente il proprio agire alle informazioni che sono fornite su di essa. E le misurazioni statistiche servono a fabbricare il futuro. È pertanto necessario interrogarsi sulla presunta oggettività dei dati, in quanto la questione della neutralità degli algoritmi con cui li trattiamo, estraendone correlazioni, è quanto meno controversa. A sostegno di questa posizione di Cardon possiamo citare ciò che ci dice una teoria fisica consolidata come la meccanica quantistica: misurare significa fissare indicatori e categorie, definire un orizzonte di attese, intervenire sulla realtà oggetto di studio e modificarla. C'è una distanza irriducibile tra descrivere e misurare: mentre la descrizione prescinde dalla dimensione temporale, dal "qui e ora", e nell'ambito di essa il soggetto non ha un ruolo di effettiva partecipazione e incidenza, la misura, al contrario, implica un'azione irreversibile. Ed è questa azione irreversibile dell'osservatore che porta a un'informazione - intesa quale esito di un'azione che dà forma all'oggetto di studio. D'altra parte, per definizione, ogni azione richiede tempo ed energia (nella fisica quantistica l'azione è un concetto fondamentale; essa è espressa, com'è noto, dal prodotto di un'energia per un tempo e si misura in unità della costante di Planck, $\mathrm{h}=6,6 \cdot 10^{-34} \mathrm{~J} \cdot \mathrm{s}$ ). Per passare da una descrizione (forma) a una misura attiva (evento) occorre dunque rimuovere la stazionarietà ed entrare nella dimensione tempo. 


\section{Termini teorici e termini osservativi}

Non è del resto la prima volta che vengono avanzate posizioni à la Anderson. Com'è noto il Circolo di Vienna aveva proposto una rappresentazione del linguaggio delle teorie scientifiche, ad esempio della fisica, basata sulla distinzione tra due classi:

- Una classe $O$ di espressioni che designano cose e proprietà considerate come "osservabili" in un qualche senso che si sia assunto per questo termine (ad esempio, 'filo di rame', 'verde' e 'più lungo di');

- e una classe $T$ di espressioni considerate come teoriche (ad esempio 'elettrone' o 'onda luminosa').

Secondo i neopositivisti per stipulazione ogni espressione non logica del linguaggio $L$ della fisica (come 'se-allora', 'non' e 'per ogni $x$ ') deve appartenere a una delle due classi $O$ e $T$. Inoltre, come precisa Nagel:

è supposto che $L$ sia un "sistema formale", cioè che soddisfi un dato numero di condizioni che di fatto non sono soddisfatte dal linguaggio corrente della fisica. In primo luogo, il vocabolario di $L$ è interamente specificato, e sono stabilite delle regole esplicite per costruire enunciati con tale vocabolario. Un enunciato le cui espressioni componenti non logiche appartengono tutte ad $O$ è chiamato un "enunciato osservativo"; un enunciato che contenga almeno un'espressione appartenente a $T$ viene detto "teorico". In secondo luogo, le inferenze lecite entro $L$ sono codificate in un insieme fissato $R$ di regole di inferenza logica. In terzo luogo, $L$ è assiomatizzato, nel modo reso familiare dalla geometria (Nagel 1961 [1968], 143).

Questo riferimento alla classica opera di Nagel consente di esplicitare quale sia l'idea-guida di Anderson: quella di sostituire un sistema linguistico formale contenente espressioni teoriche con un altro sistema formale che non abbia termini teorici e che pure abbia lo stesso contenuto empirico del sistema iniziale. In questo modo nel linguaggio figurerebbero esclusivamente termini osservativi, in grado di parlare da soli, senza bisogno di alcuna teoria per interpretarli e di alcun modello per rappresentarli.

Nagel richiama a proposito del tentativo di ridurre tutti i termini teorici a termini osservativi un teorema dimostrato da Craig nel 1953 (Craig 1953) e da lui stesso enunciato in forma meno tecnica tre anni dopo (Craig 1956) che

mostra come costruire un linguaggio formale $L^{*}$ nel seguente modo: le espressioni non logiche di $L^{*}$ sono i termini osservativi $O$ di $L$; le regole di inferenza $R^{*}$ di $L^{*}$ sono le stesse $R$ (salvo modificazioni non essenziali); i soli enunciati non logicamente veri inclusi negli assiomi $A^{*}$ di $L^{*}$ sono enunciati osservativi, specificati con una procedura effettiva eseguita sugli enunciati osservativi veri $W_{o}$ di $L$. Si può quindi dimostrare che un enunciato osservativo $S$ è un teorema di $L$ se, e solo se, $S$ è un teorema di $L^{*}$, così che il contenuto empirico di $L^{*}$ è lo stesso di 
quello di $L$. Di conseguenza, qualsiasi sistematizzazione di enunciati osservativi si raggiunga in $L$ con l'aiuto di teorie, essa appare raggiungibile in $L^{*}$ senza teorie. Sembra dunque che, dal punto di vista della logica formale, le teorie non siano strumenti essenziali per l'organizzazione della fisica (Nagel 1961 [1968], 144).

Anderson ha quindi ragione? Il teorema esposto non autorizza affatto una simile conclusione, come lo stesso Craig ebbe a rilevare ante litteram. Infatti esso mette in rilievo due limiti essenziali insiti nel passaggio da $L$ a $L^{*}$ : il primo consiste nel fatto che il metodo, benché mostri in qual modo possano venir effettivamente specificati gli assiomi $A^{*}$ di $L^{*}$, non garantisce che tali assiomi saranno in numero finito (a meno che la classe $W_{o}$ di enunciati osservativi veri di $L$ sia a sua volta finita). Il secondo sta nella mancata garanzia di poter specificare gli assiomi $A^{*}$ (in numero finito ma molto grande o addirittura infinito) in modo tale da rendere operativamente possibile usarli efficientemente per scopi deduttivi. Va in proposito ricordato che l'abituale assiomatizzazione delle teorie contiene, non a caso, non solo un numero finito di assiomi, ma addirittura un numero relativamente piccolo proprio per agevolare la deduzione. Commenta Nagel:

Un tal insieme di assiomi per $L^{*}$ non offre una formulazione semplificata del contenuto empirico di $L^{*}$, e in realtà si limita a riformularlo, in modo che gli assiomi non offrono alcun vantaggio rispetto a un semplice elenco di tutti gli enunciati osservativi veri. Per di più per poter specificare gli assiomi di $L^{*}$ dovremmo già sapere, ancor prima di averne tratto alcuna deduzione, tutti gli enunciati veri di $L^{*}$ - in altre parole il metodo di Craig ci mostra come costruire il linguaggio $L^{*}$ solo dopo che si sia compiuta ogni possibile indagine nell'argomento di $L^{*}$ (Nagel 1961 [1968], 145).

Le conseguenze sulla prospettiva di Anderson sono palesi e tutt'altro che innocue. La prima, messa in rilievo ancora da Nagel, è che

le teorie scientifiche sono soprattutto importanti non per la loro possibilità di essere vere, ma perché servono da guida all'indagine, alla formulazione e all'organizzazione di materie di fatti osservabili ancor prima che si sia stabilita la verità (o la probabile verità) di tutti gli enunciati osservativi (Nagel 1961 [1968], 145).

E la stessa cosa può essere detta, a maggior ragione, nel caso dei modelli, per i quali, contrariamente a quel che sostiene Anderson, la verità o la falsità non è la sola questione importante per quanto riguarda il riconoscimento della loro funzione (imprescindibile) nell'attività di ricerca.

La seconda conseguenza è, se si vuole, ancora più importante, e può essere evidenziata richiamando una distinzione fondamentale, basata sul concetto di sinonimia, cioè sulla presenza di segni diversi che hanno un'identità 
sostanziale di significato, fra i linguaggi con un dizionario circolare, o a rete, e i linguaggi con un dizionario verticale, o ad albero.

I dizionari delle lingue naturali hanno una struttura caratterizzata dalla presenza determinante della sinonimia e sono pertanto circolari, cioè non hanno una base, costituita da un insieme di termini primitivi di concetti elementari, a partire dai quali sia elaborato il successivo sviluppo logico dell'intelaiatura di concetti e definizioni. Essi sono quindi costruiti a rete, attraverso catene di sinonimia che si richiamano a vicenda e che evidenziano una struttura circolare, in maniera tale che ogni elemento agisce sul successivo, finché l'ultimo ritrasmette l'effetto al primo.

Le teorie scientifiche costruiscono invece dizionari che hanno una base di partenza ben definita, costituita dalle definizioni, attraverso gli assiomi, dei termini primitivi e dei concetti elementari, a partire dai quali si ha, in questo caso, il successivo sviluppo dell'intera intelaiatura del dizionario. L'esempio classico è quello della geometria euclidea, dove i cinque postulati forniscono le definizioni dei termini come "punto", retta" e "piano" o del concetto di parallelismo e non hanno, come pure si pensava fino all'avvento delle geometrie non euclidee, alcuna pretesa di esprimere verità "evidenti", e in quanto tali non bisognose di dimostrazione.

Quelli a dizionario verticale sono essenzialmente linguaggi formali, simbolici, che, usando regole di manipolazione logica autoconsistente di un repertorio di simboli condivisi (per significato e per uso) rendono manifesta una conclusione verificabile degli assunti di partenza: essi dunque contengono procedure certe per ricavare nuova informazione affidabile dall'informazione disponibile.

Ora la moltiplicazione all'infinito degli assiomi, conseguenza del teorema di Craig, ha, oltre alle altre già evidenziate, la spiacevole conseguenza di eliminare di fatto l'articolazione gerarchica dei linguaggi scientifici, cioè la distinzione fra un pacchetto ridotto di proposizioni che forniscono la definizione dei termini primitivi e dei concetti elementari e il corpo complessivo del dizionario. Una presenza così massiccia e ingombrante di assiomi rende superflua la funzione di "pietre angolari" dei linguaggi formalizzati, attribuita loro da Hilbert.

\section{Cosa succede se ci affidiamo ai soli dati ignorando le leggi}

Un altro aspetto della concezione di Anderson da evidenziare è il suo riferimento non solo alla possibilità, ma anche all'efficacia di un apprendimento di tipo statistico che avviene in assenza di una reale comprensione dei fenomeni.

Assumiamo dunque (cosa che non sempre è vera) di sapere che il sistema che vogliamo studiare è regolato da leggi deterministiche che però ci sono ignote. 
La domanda alla quale rispondere è se, in questo caso, disponendo di una grande quantità di dati che descrivono l'evoluzione del sistema, analizzandola e imparandola, si possano comprendere caratteristiche del sistema utili a conoscere il suo stato in un tempo futuro; cioè utili per eseguire una previsione affidabile.

L'idea essenziale è applicare a questi dati il cosiddetto "metodo degli analoghi", che permette, dalla conoscenza dello stato del sistema, fino a un tempo abbastanza remoto nel passato, di inferire lo stato futuro: se nella serie temporale che descrive la passata evoluzione si trova una situazione simile a quella attuale, si può sperare di apprendere qualcosa sul futuro del sistema anche in assenza di un modello che ne descriva l'evoluzione.

Il matematico polacco Mark Kac ha però mostrato che il tempo medio di ritorno in un insieme $A$ è inversamente proporzionale alla probabilità, indicata con $P(A)$, che il sistema durante la sua evoluzione si trovi in $A$. Detto altrimenti, questo tempo medio di ritorno cresce esponenzialmente con la dimensionalità del sistema stesso, ovvero con il numero di variabili rilevanti che ne descrivono lo stato fisico (Vulpiani 2013). In pratica, le regolarità in un sistema con alta dimensionalità (un sistema cioè con un numero sufficientemente grande di corpi in interazione) appaiono su scale di tempo che sono e rimarranno inaccessibili per quanto le banche dati digitali possano auspicabilmente crescere (Cecconi, Cencini, Falcioni \& Vulpiani 2012).

Nonostante un sistema sia regolato da leggi deterministiche note, è dunque possibile fare delle predizioni sulla sua evoluzione nel lungo periodo solo per dei periodi di tempo determinati dalle caratteristiche del sistema stesso. La legge di gravità che muove i pianeti, così come le leggi della fluidodinamica che descrivono la dinamica dell'atmosfera (o le leggi dell'elasticità che regolano i movimenti delle placche terrestri e dunque sono alla base dei terremoti), sono tutte leggi della fisica ben note, definite "leggi deterministiche". Malgrado questo, poiché i sistemi cui si applicano sono composti di molti corpi, esiste comunque un orizzonte di predicibilità, un tempo oltre il quale non è possibile fare una previsione affidabile perché il sistema diventa caotico. Nel caso dei terremoti, ad esempio, non è possibile conoscere lo stato del sistema a un certo tempo, se non in maniera molto grossolana, ed è dunque impossibile compiere previsioni affidabili.

\section{4. È un problema di decisione, non di sola previsione}

L'alternativa tra le due opzioni in campo può dirsi a questo punto ben delineate. Nell'approccio dei big data la funzione dei modelli e delle teorie, se non obliterata del tutto, è fortemente ridimensionata, nel senso che essi, anziché costituire delle voci di entrata nel calcolo, con funzione di indirizzo e di guida rispetto a esso, diventano voci di uscita, ex post. 
Questo approccio, proprio per la sua natura intrinseca, è esposto a un'insidia ben precisa, determinata dal fatto che, per un verso, esso presuppone ed esige la raccolta del maggior numero di dati possibile per analizzare il fenomeno a cui si è interessati; d'altro canto, però, questo orientamento provoca un'ovvia ridondanza, con inevitabile incremento del rumore dal punto di vista dell'informazione e conseguente necessità di "pulizia" e selezione dei dati medesimi allo scopo di estrarne conoscenza e disporre di informazioni in grado di supportare conclusioni e decisioni. Si riaffaccia così, alla finestra, quell'esigenza di capacità interpretativa e di ricerca del "perché" di determinati fenomeni emergenti, precedentemente cacciata dalla porta. Anche ammesso che i dati, in prima istanza, "parlino da soli", senza bisogno di particolari chiavi interpretative, la questione che emerge è quella riguardante la loro capacità di rispondere, sempre da soli, alla necessità di caratterizzarsi e classificarsi sulla base di un criterio di pertinenza che possa fungere da strumento operativo affidabile per poter compiere una selezione interna ragionevole ed efficace.

Il processo che porta a selezionare i dati e le informazioni da considerare rilevanti ai fini delle decisioni da prendere rischia di essere un circolo vizioso se non si spiega quali sono i fattori in base ai quali viene operata questa scelta e non si mostra in modo convincente che essi non richiedono, ancora una volta, null'altro che non sia riconducibile al puro e semplice accumulo dei dati medesimi e alla loro gestione algoritmica. Questo problema è acuito dal fatto che le smart technologies permettono la convergenza e lo scambio dialogico di domini diversi, che precedentemente e in altri contesti non avrebbero mai avuto la possibilità di interagire. La scrittura e la lettura del mondo attraverso i dati richiede di conseguenza una capacità descrittiva e di analisi di sistemi complessi in interazione reciproca tale da far emergere l'esigenza imprescindibile di ricomporre e integrare in aggregati di sempre maggiore complessità frammenti di conoscenza prima spezzettati e sparsi in ambiti differenti. È proprio questa interazione di domini diversi - fisici, biologici e digitali - a caratterizzare la quarta rivoluzione industriale e a renderla diversa dalle rivoluzioni precedenti.

Il quadro che ne scaturisce dilata le possibilità di innovazione ma aumenta l'esigenza di interdisciplinarità e di integrazione delle conoscenze per gestirla:

Oltre alla velocità e alla capacità di diffusione, la quarta rivoluzione industriale è unica in considerazione della crescente armonizzazione tra le diverse discipline e innovazioni. Si tratta di innovazioni concrete, che derivano dalla interdipendenza di diverse tecnologie e che non appartengono più soltanto al mondo della fantascienza. Oggi, per esempio, le tecnologie per la fabbricazione digitale possono interagire con la dimensione biologica. Alcuni design e architetti stanno già combinando aspetti di design computazionale, processi additivi, ingegneria dei materiali e biologia di sintesi per elaborare sistemi che favoriscono l'integrazione fra microrganismi, il nostro 
corpo, i prodotti che consumiamo, e addirittura gli edifici in cui viviamo. (Schwab 2016, 25).

Questa crescente dilatazione e integrazione della conoscenza va gestita e per farlo non ci si può affidare alla sola previsione, cioè al semplice desiderio di "sapere come le cose andranno... come se andassero per conto loro!". Si tratta "di un problema di decisione, non di previsione", come ha avuto il grande merito di sottolineare Bruno de Finetti (2006) nel suo agilissimo e lucidissimo saggio del 1934 L'Invenzione della Verità (scritto a 28 anni), splendido esempio di concezione costruttivista della scienza, un antidoto contro ogni cristallizzazione in "verità assolute" del territorio mobile della conoscenza. Questo è il punto: per l'uomo la previsione non è un fine, ma un mezzo, uno strumento per assumere decisioni efficaci, per cui è necessario abbandonare l'idea unidimensionale di "previsione" per passare a quella ben più complessa di "strategia", basata sul ruolo attivo dell'osservatore. Ovviamente un passaggio di questo genere è perfettamente compatibile con gli approcci che pongono al centro della propria riflessione l'idea di progetto, in coerenza con gli sviluppi delle neuroscienze sui quali abbiamo concentrato l'attenzione, mentre non lo è con le prospettive à la Anderson, che negano, come si è visto, l'importanza delle teorie e dei modelli e disconoscono il ruolo del soggetto osservante. Qui sta la netta linea di demarcazione tra le due posizioni che intendiamo rilevare. Essa infatti evidenzia come quella che possiamo definire la pratica di costruzione degli scenari futuri sia qualcosa di profondamente diverso dagli scenari in sé, dato che il suo obiettivo non è solo né prioritariamente l'analisi finalizzata alla previsione, ma piuttosto l'elaborazione del senso e della conoscenza che i soggetti partecipanti alla costruzione di questi scenari devono saper esprimere per mobilitarsi in modo coeso e coordinato a un'azione che sia in grado di rendere antifragile il loro paesaggio.

Il concetto di antifragilità, proposto di recente da Taleb (2012) per caratterizzare i sistemi capaci di "prosperare nel disordine", va oltre l'idea di "resilienza" in quanto, a differenza di quest'ultima, non denota la capacità dei sistemi medesimi di resistere agli shock, rimanendo gli stessi di prima, bensì la proprietà di uscire migliorati da questo confronto con la casualità, l'incertezza e il caos, come fa tutto ciò che sa cambiare nel tempo crescendo: l'evoluzione, la cultura, le idee vincenti, i buoni sistemi politici, l'innovazione tecnologica. Fare riferimento a questo concetto significa dunque porsi l'obiettivo di un ridisegno dell'ambiente istituzionale nel quale si vive tale da metterlo in condizione di uscire irrobustito dall'imprescindibile inserimento nell'evoluzione delle economie globali, con il portato di impatto in termini socioeconomici per la dimensione locale che esso comporta.

Un problema di questa portata per quanto riguarda i contesti territoriali, si tratti di città o più in generale di paesaggi, non può essere affrontato con l'ausilio delle sole tecnologie o degli algoritmi, né può basarsi su metodi che fanno affidamento esclusivamente sulle previsioni o sulla possibilità di 
estrarre dai dati il benchmark significativo, la correlazione importante e pertinente. Come infatti sottolinea Carlo Ratti, direttore del Laboratorio Senseable City del MIT, pur dando il corretto rilievo all'importanza di questi fattori, non si può pensare seriamente di venirne a capo senza fare riferimento alla smartness dei cittadini e di coloro che abitano il paesaggio:

l'esclusivo focus sull'efficienza non tiene conto dei fondamentali scopi civici, come la coesione sociale, la qualità della vita, la democrazia e lo stato di diritto. Sviluppare la socialità con la tecnologia è, tuttavia, diretto a questa esigenza, mentre allo stesso tempo apre a nuovi approcci verso l'efficienza (Ratti \& Townsend 2014).

Rendere sempre più intelligenti le città e sempre più adeguati alle sfide del nostro tempo i paesaggi, senza per questo snaturarli e privarli della loro identità, frutto dell'azione di fattori naturali, umani e delle loro interrelazioni, presuppone ed esige il riferimento a capacità progettuali, a una pratica $d i$ costruzione degli scenari futuri da riuscire prima a immaginare, anticipando l'esperienza, e poi a realizzare, assoggettandoli alla propria capacità creativa. Questa pratica di costruzione degli scenari futuri è assimilabile alle competenze del cartografo, il quale sa bene che l'efficacia di una mappa nel rappresentare il territorio è data dalla capacità di riuscire a bilanciare il più possibile le tre seguenti coppie:

- sintetico - analitico;

- globale - funzionale;

- comprensivo - selettivo.

Non è possibile rappresentare il mondo o una sua porzione in una carta come suo doppio. La carta è un modello, non la realtà, e per elaborarla occorre un procedimento di riduzione e selezione, perché una mappa, come quella dell'impero di Borges, che coincida con quest'ultimo sarebbe del tutto ridondante e inutile. Ogni volta che si realizza una carta è quindi necessario operare prcise scelte progettuali, in funzione degli scopi che si intendono perseguire, come appunto ci dicono le neroscienze. Queste scelte riguardano, ad esempio, la proiezione cartografica da adottare, quella concernente il tematismo e quindi il contenuto della carta, quella dipendente dalla scala di rappresentazione scelta ecc.

La carta non si limita dunque a riprodurre il mondo, ma lo costruisce, ed è proprio in questo senso che si parla di pratica di costruzione degli scenari. Se è la mappa che crea ciò che rappresenta, rendendolo comprensibile $\mathrm{e}$ utilizzabile in modo efficace, bisogna concludere che, prima della mappa, non sussiste alcun significato già dato: è solo in virtù di essa che la natura intangibile del dato diventa accessibile, interpretabile e utilizzabile.

Anche se nella spiegazione dei fenomeni si ricercano correlazioni invece che cause non è dunque possibile procedere semplicemente gettando i numeri in 
un grande cluster di calcolo e lasciare che gli algoritmi trovino modelli laddove la scienza non può. Per passare dal dato alla conoscenza occorre un metodo in grado di considerare le diverse variabili e implicazioni che intervengono nei processi conoscitivi e che sono esterni al dataset.

Tutto ciò significa che per evitare di cadere in un circolo a vizioso occorre spostare il baricentro delle ricerche e delle applicazioni riguardanti l'intelligenza artificiale dal sapere maturo ai processi di apprendimento. Ciò vuol dire, concretamente, due cose ben precise. In primo luogo che il traguardo che la macchina deve raggiungere non è più l'esperto che dispone già di conoscenza d'alto profilo e la sa usare, ma il bambino che impara e l'itinerario attraverso il quale egli riesce ad accrescere via via ciò che sa. In secondo luogo che la via da seguire per riprodurre questo processo non è più basata sull'accoppiamento tra insegnamento e apprendimento ma sulla loro scissione, a vantaggio di quest'ultimo. Anziché avere un "docente" che "istruisce" la macchina mostrando a essa una quantità enorme di esempi della forma da riconoscere e correggendola allorché sbaglia, finché non riesce a eseguire correttamente il compito che le è stato assegnato, la macchina deve riuscire ad apprendere in modo autonomo, senza essere programmata espressamente e guidata. L'espressione di questa svolta è il concetto di unsupervised machine learning, l'apprendimento senza tutore, in cui il computer ha le domande ma non le risposte e deve trovare da solo come arrivare a queste ultime.

Per "centrare" questo obiettivo si analizzano in particolare i meccanismi del linguaggio e della visione e per rimarcare l'analogia con la struttura del cervello umano si parla di reti neurali e si cerca di riprodurre il modo in cui certi stimoli attivano determinate connessioni tra neuroni. La meta da raggiungere, fissata da alcuni come Ray Kurzweil, informatico e inventore statunitense, pioniere nei campi del riconoscimento ottico dei caratteri e delle tecnologie sulla decodificazione del parlato, è la singolarità tecnologica, il momento in cui i computer saranno in grado da soli di progettare e costruire altre macchine, via via più intelligenti in un processo di crescita e di selezione artificiale inarrestabile, in grado di superare, per velocità ed efficienza, la selezione naturale. Data fissata per questo traguardo il 2045. Risultato previsto che affascina e nello stesso tempo turba il grande Stephen Hawking, secondo il quale riuscire a creare un'intelligenza artificiale sarebbe l'evento più grande della storia dell'uomo. Purtroppo però potrebbe anche essere l'ultimo. Se l'evoluzione naturale ha prodotto il passaggio cruciale dalla scimmia all'uomo, l'evoluzione artificiale, creando un'intelligenza delle macchine superiore a quella umana, potrebbe provocare la retrocessione dell'uomo, rispetto a questo nuovo stadio evolutivo, al rango di scimmia.

Il traguardo della singolarità tecnologica è pertanto un presupposto imprescindibile se si vuole trasformare il motore dell'approccio conoscitivo basato sui big data in un circolo virtuoso capace di alimentarsi di continuo e di 
diventare capace di selezionare ogni volta i dati e le informazioni da ritenere rilevanti per le decisioni. Per riuscire ad analizzare enormi quantità di dati in maniera meccanica è indispensabile che l'algoritmo sia in grado di apprendere ogni volta dall'esperienza pregressa, riuscendo così a migliorare la sua performance nelle successive analisi che dovrà compiere. Ma, come sottolinea Pedro Domingos in un recente volume riguardante il machine learning (Domingos 2015 [2016, 299]) anche se questo campo da ricerca ha avuto negli ultimi anni un notevole sviluppo i problemi ancora da risolvere verso un possibile algoritmo definitivo sono ancora notevoli. Un simile algoritmo, infatti, dovrebbe essere più interattivo, avere una capacità introspettiva ed essere in condizione di spiegare il proprio ragionamento. Dovrebbe sostanzialmente riuscire a incorporare anche quelle variabili che incidono in maniera rilevante nei processi conoscitivi e che sono esterni al dataset.

Proprio questo è l'oggetto della controversia tra catastrofisti e innovatori: gli uni e gli altri condividono lo stesso presupposto: l'inarrestabilità del processo in atto e degli sviluppi previsti. Mentre però i primi sembrano rassegnati a subirne gli esiti, i secondi cercano di capire come, a sua volta, l'intelligenza dell'uomo possa migliorarsi e accelerare il proprio processo di sviluppo traendo tutti i possibili insegnamenti proprio dall' unsupervised machine learning.

A sorreggere questa loro speranza vi è un fatto ben preciso, attestato, ancora una volta, dai risultati delle neuroscienze: l'accelerazione sempre più rapida, proprio in seguito all'interazione con i progressi della tecnologia, del ritmo dell'evoluzione naturale, in seguito alla quale nel passaggio ormai da una generazione all'altra il cervello mostra cambiamenti che precedentemente avrebbero richiesto tempi assai più lunghi. È dunque sbagliato, a loro giudizio, concentrare l'attenzione solo sugli sviluppi dell'intelligenza artificiale e sul loro ritmo rimanendo ancorati alla concezione tradizionale secondo la quale l'evoluzione naturale ha un andamento proprio che non può essere velocizzato.

Ecco perché è importante non lasciarsi spaventare e distogliere dalle voci insistenti e assordanti di coloro per i quali la tecnica è l'origine di tutti i mali, la causa principale dell'alienazione e frustrazione degli uomini, lasciandosi invece guidare dall'idea che è proprio in quello che viene ritenuto un pericolo che spesso si annida ciò che salva.

Ecco il punto. Gli innovatori, quelli veri, si muovono sulle tracce di Chirone, il centauro rimasto coinvolto nella battaglia tra i suoi simili ed Ercole e colpito a una gamba da una freccia avvelenata scoccata dal nemico. Egli non riuscì mai più a guarire da quella ferita, ma anziché cercare di rimuoverla o lasciare che contaminasse il resto del suo corpo, ne fece uno strumento di conoscenza diretta delle piaghe e della malattia e acquisì una tale maestria nell'arte del curare da diventare mentore di Ippocrate. Essi dunque non negano che la tecnologia possa essere un vulnus, una hỳbris, espressione di un atteggiamento 
di "insolenza e tracotanza" nei confronti della natura, con l'obiettivo di piegarla e domarla, e dunque manifestazione di un orgoglio che può portare all'ostinata sopravvalutazione delle proprie forze e alla prevaricazione. Ma, come Chirone, si rendono conto che la cura non può consistere nel cancellarla dalla nostra vita, obiettivo del tutto utopistico e impraticabile, ma nel trasformarla invece in terapia in grado di sanare le distorsioni da essa stessa provocate.

Anziché respingerla, ne fanno per questo oggetto di un pensiero riflessivo e critico, fino a individuare il circolo vizioso che è alla base di tutti i suoi mali: l'interesse esclusivo per l'uso e l'utile, che genera la riduzione del pensiero a un apprendimento di tipo statistico che può avvenire anche in assenza di una reale comprensione dei fenomeni, ad algoritmi autosufficienti al punto da ritenere che non si abbia bisogno di altro per comprendere i fenomeni naturali e sociali.

Questo tipo di pensiero calcolante, che punta a rimpiazzare la libera iniziativa dell'uomo con un procedimento meccanico, che può essere compiuto anche da una macchina, pur indispensabile per conferire rigore alla conoscenza, non lascia spazio alla meraviglia, allo stupore, all'immaginazione, all'esplorazione di alternative, alla creatività, e si concentra unicamente sulla capacità di previsione e sull'applicazione dell'esito al quale si perviene. Viene allora sacrificata la natura sociale e interattiva della conoscenza, quella che ne fa l'agente propulsivo di una spirale virtuosa che parte dalla libera espressione ed emanazione di una comunità e dalle sue acquisizioni, che diventano fonte di un legame sociale sempre più forte e duraturo, in quanto ne scaturisce una visione comune, uno sfondo condiviso di ideali e valori.

Questi ideali e valori non possono però restare solo enunciazioni teoriche astratte: vanno trasformati in obiettivi concreti da realizzare, e per farlo occorre agire tecnicamente. La tecnologia deve per questo diventare vissuto, esperienza, forma di vita in grado di aprire la strada, nella società, a una formidabile disposizione creativa, attraverso la quale l'uomo meditante, e non semplicemente calcolante, capisce di poter abitare una pluralità di ambienti differenti, tanti quanto sono i contesti tecnici e i media che è capace di creare. La sfida da affrontare diventa allora quella di radicare i nuovi ambienti così prodotti nel vissuto quotidiano, con una nuova alleanza tra artificiale e naturale che innalzi il livello qualitativo dell'esperienza comune dando vita a nuove forme di interazione e di condivisione tra gli uomini, sempre più efficaci e durature.

Ecco allora la terapia individuata dagli innovatori, novelli Chironi, per guarire la tecnologia dalle ferite che l'inquinano: trasformarla da fonte di alienazione in forza capace di esaltare l'immaginazione e il carattere interattivo della ricerca, orientandola verso idee e scoperte grazie alle quali si possa incrementare e rafforzare la cultura partecipativa. Portarla a un livello di disposizione creativa e di diffusione capillare così elevate da far capire a tutti 
che, agendo tecnicamente e valendoci delle opportunità offerte dalle reti, possiamo cooperare anziché limitarci a competere, rendendoci conto che come non possiamo affatto concepire oggetti spaziali fuori dello spazio e oggetti temporali fuori del tempo, così non possiamo realmente pensare alcun uomo, soggetto sociale per definizione, fuori della possibilità della sua relazione con altri, fuori da uno spazio comunitario.

Non si tratta di una sterile utopia: è invece un comportamento sociale codificato, alla base del quale vi è un concetto ben preciso, messoci a disposizione proprio dal pensiero calcolante, quello di gioco a somma positiva, in cui i giocatori coinvolti vincono o perdono insieme. A esprimere questo concetto in modo tanto concreto quanto mirabile è stato John Nash (1996), il matematico ed economista statunitense, tra i più brillanti e originali del Novecento, la "mente meravigliosa" dell'omonimo film, al quale fu assegnato, per questa scoperta, il premio Nobel per l'economia nel 1994. È importante capire bene questo tipo di giochi, differenziandoli da quelli a somma zero, i quali descrivono una situazione nella quale il guadagno o la perdita di un giocatore è perfettamente bilanciato da una perdita o da un guadagno di un altro, per cui se dalla somma totale dei guadagni di tutti i protagonisti si sottrae la somma totale delle perdite si ottiene appunto, zero.

La nascita della moderna teoria dei giochi viene generalmente fatta coincidere con l'uscita del libro Theory of Games and Economic Behavior di John von Neumann e Oskar Morgenstern nel 1944, anche se altri autori, quali Ernst Zermelo, Armand Borel e lo stesso von Neumann avevano anticipato l'idea di descrivere matematicamente ("matematizzare") il comportamento umano in quei casi in cui l'interazione fra contendenti comporta la vincita o la suddivisione di un qualche tipo di risorsa.

La premessa indispensabile è che tutti devono essere a conoscenza delle regole del gioco, ed essere consapevoli delle conseguenze di ogni singola mossa. Nella teoria la mossa, o l'insieme delle mosse che un individuo intende fare, viene chiamata "strategia". Ogni giocatore può prendere un numero finito (o infinito nel caso più astratto possibile) di decisioni o strategie. Ogni strategia è caratterizzata da una conseguenza per il giocatore che l'ha presa, che può essere un premio o una penalità quantificabili.

Il risultato del gioco è completamente determinato dalle sequenze delle rispettive strategie. Si può rappresentare ogni gioco con un grafo ad albero che descrive ogni possibile combinazione di giocate dei contendenti sino agli stati finali, nei quali vengono ripartite le vincite. Questa descrizione è quella che viene immediatamente in mente per esempio quando si pensa al gioco degli scacchi. A ogni mossa di uno dei giocatori possono seguire più risposte dell'altro, a ognuna delle quali a sua volta il primo può rispondere scegliendo fra parecchie mosse possibili, e così via. La struttura che descrive questa situazione è appunto la struttura di un albero, nel quale ogni ramo si divide in più rametti, i quali a loro volta si dividono in rametti più sottili e così via. 
Il contributo fondamentale di Nash consiste nell'introduzione della nozione di "equilibrio", grazie alla quale la possibilità di applicare la Teoria dei Giochi ai casi concreti di conflitto ha compiuto un passo avanti decisivo. Questa scoperta, che gli valse il premio Nobel per l'economia nel 1994, prende in considerazione, in particolare, un comportamento che non può essere migliorato con azioni unilaterali, nel senso che lo si sarebbe tenuto anche se il comportamento dell'avversario fosse stato noto in anticipo. È stato lo stesso John Nash a esporre in modo sintetico ed efficace, in una intervista rilasciata nel 2015, poco prima della morte, le basi della sua teoria, facendo emergere, come tratto distintivo fondamentale del suo approccio, l'importanza della cooperazione tra i giocatori: "Un gioco può essere descritto in termini di strategie che i giocatori devono seguire nelle loro mosse: l'equilibrio c'è quando nessuno riesce a migliorare in maniera unilaterale il proprio comportamento. Per cambiare, occorre agire insieme". E ancora: "Unilateralmente possiamo solo evitare il peggio, mentre per raggiungere il meglio abbiamo bisogno di cooperazione".

Assumendo il gioco a somma positiva come schema per esemplificare le relazioni sociali ciò che si vuole mettere in evidenza è che i soggetti coinvolti sono interessati a interagire e a sostenersi reciprocamente, stabilendo un concreto rapporto di fiducia e collaborazione, accrescendo la riserva strutturale di opportunità complessive sulla quale il sistema sociale e l'ambiente in cui sono inseriti e operano possono contare.

È questo il significato fondamentale del passaggio dai giochi a somma zero, nei quali non si ha mai incremento di valore, dato che il risultato che si ottiene sottraendo dalla somma totale dei guadagni di tutti i protagonisti la somma totale delle perdite è sempre uguale a zero, ai giochi a somma positiva.

Questo passaggio rende possibile l'emergere di quello che Heinz von Foerster considera l' imperativo etico" fondamentale del nostro tempo, che invita a un'azione orientata a produrre nuove possibilità per sé stesso e per il prossimo: "agisci sempre in modo di accrescere il numero totale delle possibilità di scelta", "do ut possis dare", do affinché tu possa dare di più (Von Foerster 1982, 233). Seguendo questa traccia ci si orienta verso una strategia di continua creazione di possibilità nella quale ogni decisione, ogni azione, ogni comportamento, attualizza una parte del possibile mentre crea un nuovo possibile. Non, quindi, il possibile in modo generico e indeterminato, come risultato dell'esclusione di ciò che è necessario e ciò che è impossibile, ma il possibile come l'inserimento di ciò che è dato nell'orizzonte delle sue possibili trasformazioni, concepibili e concretamente realizzabili. Un confine, dunque, nel quale inserire la crescita e lo sviluppo, per evitare, come si è avuto già occasione di dire, che essi vengano considerate prospettive incondizionate e senza limiti.

L'imperativo di von Foerster è importante perché coglie ed esprime la tendenza fondamentale sia della vita, sia della conoscenza, in virtù del forte 
legame che viene sempre più istituito tra di esse, che vogliono continuamente sperimentarsi, espandersi, calpestare le frontiere, ridurre le terre di nessuno. La "vita vivente" e il processo della conoscenza vogliono proprio questo. Imprevedibilità, invenzione, di conseguenza, vanno accettate e coltivate con attenzione, garantendo a esse l'indispensabile ancoraggio alla realtà esistente, al costante confronto con la quale non ci si può, ovviamente, sottrarre.

\section{Conclusione: è un problema di democrazia}

Dalla comparazione delle due prospettive di ricerca prese in esame emerge con chiarezza un aspetto che rende quanto mai attuale e profetico il seguente passo di Gramsci:

È da notare che accanto alla più superficiale infatuazione per le scienze, esiste in realtà la più grande ignoranza dei fatti e dei metodi scientifici, cose molto difficili e che sempre più diventano difficili per il progressivo specializzarsi di nuovi rami di ricerca. La superstizione scientifica porta con sé illusioni così ridicole $\mathrm{e}$ concezioni così infantili che la stessa superstizione religiosa ne viene nobilitata. Il progresso scientifico ha fatto nascere la credenza e l'aspettazione di un nuovo tipo di Messia, che realizzerà in questa terra il paese di Cuccagna; le forze della natura, senza nessun intervento della fatica umana, ma per opera di meccanismi sempre più perfezionati, daranno alla società in abbondanza tutto il necessario per soddisfare i suoi bisogni e vivere agiatamente. Contro questa infatuazione, i cui pericoli sono evidenti (la superstiziosa fede astratta nella forza taumaturgica dell'uomo, paradossalmente porta ad isterilire le basi stesse di questa forza e a distruggere ogni amore al lavoro concreto e necessario, per fantasticare, come se si fosse fumato una nuova specie di oppio) bisogna combattere con vari mezzi, dei quali il più importante dovrebbe essere una migliore conoscenza delle nozioni scientifiche essenziali, divulgando la scienza per opera di scienziati e di studiosi seri e non più di giornalisti onnisapienti e di autodidatti presuntuosi. In realtà, poiché si aspetta troppo dalla scienza, la si concepisce come una superiore stregoneria, e perciò non si riesce a valutare realisticamente ciò che di concreto la scienza offre (Gramsci 1975, vol. I, 513-514).

Sembra una critica ante litteram della nuova mitologia di cui Anderson si fa portabandiera: Gramsci ci insegna che bisogna smascherare la presunta oggettività dei dati e la pretesa di poter ricavare correlazioni e informazioni significative facendo esclusivamente affidamento su di essi.

Vi è poi un altro aspetto da sottolineare per quanto riguarda la supposta oggettività del paradigma big data, per smascherare la quale la lettura di molte pagine di Gramsci risulta salutare. La prima è che i grandi filoni di dati non sono a disposizione di chiunque, ma tendono a essere oggetto di un controllo 
oligopolistico. Pochi operatori, a livello mondiale, detengono e organizzano la maggior parte dei dati che ci riguardano, per lo meno per quanto concerne la nostra vita online. Parliamo soprattutto di Google, Facebook e Amazon, i signori dei big data. Del resto solo loro dispongono dell'infrastruttura e della potenza di calcolo necessarie per effettuare analisi su tali dati. Ed è chiaro che tali soggetti agiscono sulla spinta di interessi commerciali tutt'altro che neutrali. Si aggiunga il fatto che le caratteristiche degli algoritmi utilizzati da Google, Facebook e Amazon non ci sono note, in quanto parte di un segreto industriale ben custodito. In questo senso va segnalato un bel saggio di Frank Pasquale (2015), nel quale si parla di big data come "scatole nere" e si contrappone la sempre maggiore trasparenza e apertura delle nostre vite private a chi ha la possibilità di raccogliere tutte le informazioni, come password, contenuti, nomi, numeri di carte di credito che trasmettiamo ogni giorno, al velo di segretezza che caratterizza molte architetture dei sistemi digitali, che rende estremamente opaco il meccanismo delle aziende alle quali consegniamo quei dati. Ad esempio per quanto sulla carta il meccanismo di Google appaia piuttosto chiaro, in quanto i risultati sono ordinati in base alla qualità dei contenuti dei siti e al numero e alla bontà dei link che puntano verso i siti stessi, il suo algoritmo resta rigorosamente segreto. In genere funziona, ma non abbiamo modo di sapere nel dettaglio come funziona. In sintesi, le black box creano delle aziende in cui le economie sono separate e disuguali, emergono e si rafforzano poteri invisibili fondati sulla segretezza legalizzata e su algoritmi opachi. Ecco perché secondo Pasquale è importante rendersi conto che i nostri dati sono una fonte di grande profitto per altri, spesso a nostre spese, e se finiscono nelle mani sbagliate possono costarci cari. Per scongiurare questi rischi e gli abusi che ne possono derivare occorre lavorare per una società più trasparente, senza per questo far diventare un feticcio la trasparenza assoluta, che diventerebbe un incubo di segno opposto. L'autore propone invece una trasparenza qualificata, dove la rivelazione delle informazioni è fatta rispettando gli interessi delle persone coinvolte e invita a lottare per una società intelligibile, dove la conoscenza sia distribuita e non vi siano scatole nere studiate su misura per proteggere gli interessi di pochi.

Analisi di questo tipo evidenziano che abbiamo bisogno, sempre più bisogno, di teoria, di modelli efficaci, di cultura diffusa e di chiavi interpretative: operazioni come quelle di Anderson e di molti dei suoi seguaci interessati, che parlano di "fine della teoria" appaiono, se lette con il supporto di passi come quello di Gramsci che ho citato e dell'approfondita analisi critica di Pasquale, ciò che effettivamente sono: una riproposizione in chiave moderna ed efficientistica della classica (e oscurantistica) teoria della "doppia verità", che mira a privare il senso comune della possibilità e del diritto di capire, riservato a élite sempre più ristrette e potenti. 
Si tratta di un approccio assai pericoloso, proprio per la natura stessa della democrazia, che costituisce il suo "marchio di fabbrica" esclusivo, quando è vera: essa, come sottolinea Castoriadis,

è quel regime che rinuncia esplicitamente a ogni garanzia ultima e che non conosce altra limitazione che la sua autolimitazione. Ovviamente, essa può trasgredire questa autolimitazione, come è spesso successo nella storia, e può quindi inabissarsi o ribaltarsi nel suo contrario. Ciò significa che la democrazia è il solo regime politico tragico, è il solo regime che rischia, che affronta apertamente la possibilità della propria autodistruzione (Castoriadis 1986, 71).

Per questo, per sopravvivere e per uscire indenne dal pericolo insito nella propria autolimitazione, ha bisogno di una forte consapevolezza e di una conoscenza non solo robusta e rigorosa, ma capillarmente diffusa.

\section{Bibliografia}

Anderson, C. 2008. The End of Theory: The data Deluges Makes the Scientific Method Obsolete. Wired Magazine, 23 June 2008.

Black, M. 1952. Models and Metaphors, New York: Ithaca (tr. it. Modelli, archetipi, metafore. Parma: Pratiche Editrice 1983).

Cardon, D. 2016. Che cosa sognano gli algoritmi. Le nostre vite al tempo dei big data, Milano: Mondadori Università.

Castoriadis, C. 1986. La logica de los magmas y la question de la autonomia, in: Id., Dominios del Hombre: Encrucijadas del labirinto II. Barcellona: Gedisa (tr. it. La logica del magma, in L. Guzzardi, a cura di, Il pensiero acentrico, Milano: Elèuthera 2015, 41-72).

Cecconi, F., Cencini, M., Falcioni, M. \& Vulpiani, A. 2012. Predicting the Future From the Past: An Old Problem From a Modern Perspective. American Journal of Physics, 80, 1001-1008.

Craig, W. 1953. On Axiomatization within a System. Journal of Symbolic Logic, $18,30-32$.

Craig, W. 1956. Replacement of Auxiliary Expressions. Philosophical Review, $65,38-55$.

De Finetti, B. 2006. L'invenzione della verità. Milano: Raffaello Cortina.

De Mauro, T. 1982. Minisemantica dei linguaggi verbali e non verbali e delle lingue. Bari: Laterza.

Dirac, P. A. M. 1959. I principi della meccanica quantistica. Torino: Boringhieri.

Domingos, P. 2015. The Master Algorithm. How the Quest for the Ultimate Learning Machine Will Remake Our World, New York: Basic Books (tr. it. L'algoritmo definitivo. La macchina che impara da sola e il futuro del nostro mondo, Torino: Bollati Boringhieri 2016).

Edelman, G. M. 1991. Il presente ricordato. Milano: Rizzoli. 
Gallese, V. 2003. Neuroscienza delle relazioni sociali. In F. Ferretti (a cura di), La mente degli altri. Prospettive teoriche sull'autismo. Roma: Editori Riuniti.

Gibson, J. J. 1979. The Ecological Approach to Visual Perception, Boston: Houghton Mifflin (tr. it. Un approccio ecologico alla percezione visiva, Bologna: Il Mulino, 1999).

Gramsci, A. 1975. Quaderni dal carcere. Edizione critica dell'Istituto Gramsci, a cura di V. Gerratana, vol. I-IV. Torino: Einaudi.

Heidegger, M. 1962. Kant e il problema della metafisica. Milano: Silva.

Hesse, M. B. 1980. Modelli e analogie nella scienza, a cura di C. Bicchieri. Milano: Feltrinelli.

Hey, T., Tansley, S. \& Tolle, K. (eds) 2009. The Fourth Paradigm. Data-Intensive Scientific Discovery, Microsoft Corporation.

Kant, I. 1957. Critica della ragion pura. Torino: Einaudi.

Keysers, C., Wickers, B., Gazzola, V., Anton, J.-L., Fogassi, L., \& Gallese, V. 2004.

A Touching Sight: SII/PV Activation During the Observation and Experience of Touch. Neuron, 42.

Kitchin, R. 2014. The Data Revolution: Big Data, Open Data, Data Infrastructures and Their Consequences. Los Angeles - London - New Delhi - Singapore - Washington DC: Sage.

Leopardi, G. Zibaldone di pensieri. Firenze 30 novembre 1828.

Nagel, E. 1961. The Structure of Science. San Diego, California: Harcourt, Brace \& World, Inc. (tr. it. Milano: Feltrinelli, 1968).

Nash, J. 1996. Essays on Game Theory. Northampton MA, Cheltenham Camberley, UK: Edward Elgar Pub.

Pasquale, F. 2015. The Black Box Society: The Secret Algorithms That Control Money and Information. Cambridge MA: Harvard University Press.

Pietsch, W. 2015. Aspects of Theory-Ladenness in Data-Intensive Science. Philosophy of Science, 82 (5), 905-916.

Ratti, C. \& Townsend, A. 2014. The Social Nexus, in: Designing the Urban Future: Smart Cities. New York: Scientific American.

Rizzolatti, G. \& Sinigaglia, C. 2006. So quel che fai. Il cervello che agisce e $i$ neuroni specchio. Milano: Raffaello Cortina.

Schwab, K. 2016. The Fourth Industial Revolution. Ginevra: World Economic Forum, (tr. it. La quarta rivoluzione industrial, Milano: Franco Angeli 2016).

Tagliagambe, S. 2002. Il sogno di Dostoevskij. Come la mente emerge dal cervello. Milano: Raffaello Cortina.

Taleb, N. N. 2012. Antifragile: Things That Gain from Disorder. London: Allen Lane (tr. it. Antifragile. Prosperare nel disordine. Milano: Il Saggiatore, 2013).

Toraldo di Francia, G. 1976. L'indagine del mondo fisico. Torino: Einaudi.

Von Foerster, H. 1982. Sistemi che osservano, (tr. it. Roma: Astrolabio, 1987). 
Von Neumann, J. \& Morgenstern, 0. 1944. Theory of Games and Economic Behavior. Princeton NJ: Princeton Univeristy Press.

Vulpiani, A. 2013. Problemi e limiti delle previsioni. Le Scienze, 538. 
Silvano Tagliagambe

(Università di Sassari, sil.tagliagambe@gmail.com)

Costruire scenari per il futuro

\begin{abstract}
English). In 2008 Chris Anderson wrote a provocative piece titled TheEnd of Theory The idea being that we no longer need to abstract and hypothesis; we simply need to let machines lead us to the patterns, trends, and relationships in social, economic, political, and environmental relationships. According to Anderson, the new availability of huge amounts of data offers a whole new way of understanding the world. Correlation supersedes causation, and science can advance even without coherent models and unified theories. But numbers, contrary to Anderson's assertion, do not, in fact, speak for themselves. From the neuroscience's standpoint, every choice we make is a reflection of an, often unstated, set of assumptions and hypotheses about what we want and expect from the data: no assertion, no prediction, no decision making is possible without an a priori opinion, without a project. Data-driven science essentially refers to the application of mathematics and technology on data to extract insights for problems, which are very clearly defined. In the real world, however, not all problems are such. To help solve them, one needs to understand and appreciate the context. The problem of landscape becomes, for this reason, critical and decisive. It requires an interdisciplinary approach consisting of several different competencies and skills.
\end{abstract}

Keywords (English): Big Data, Model, Project, Landscape, Antifragility, Democracy

\begin{abstract}
Italian). Nel 2008 Chris Anderson ha scritto un pezzo provocatorio dal titolo TheEnd of TheoryL'idea e che non abbiamo piu bisogno di astrarre e di ipotesi; abbiamo semplicemente bisogno di lasciare che siano le macchine a individuare modelli, tendenze e relazioni nei rapporti sociali, economici, politici e ambientali. Secondo Anderson la nuova disponibilita di enormi quantita di dati offre un nuovo modo di concepire il mondo. La correlazione sostituisce la causalita, e la scienza puo avanzare anche senza modelli coerenti e teorie unificate. Ma i numeri, contrariamente a quanto affermato di Anderson, di fatto non sono in grado di parlare da soli. Dal punto di vista delle neuroscienze, ogni scelta che facciamo e un riflesso di un insieme di supposizioni e ipotesi, che spesso rimane implicito, su cio che vogliamo e ci aspettiamo dai dati: nessuna affermazione, nessuna previsione, nessun processo decisionale e possibile senza un'opinione a priori, senza un progetto. La scienza basata sull'analisi dei dati riguarda essenzialmente l'applicazione
\end{abstract}


della matematica e della tecnologia ai dati per estrarre intuizioni per problemi molto ben definiti. Nel mondo reale, tuttavia, non tutti i problemi sono tali. Per aiutarci a risolverli c'e bisogno di capire e apprezzare il contesto. Il problema del paesaggio diventa, per questo motivo, critico e decisivo. Esso richiede un approccio interdisciplinare che possa fruire di una serie di competenze e abilita diverse.

Keywords (Italian): Big Data, Modello, Progetto, Paesaggio, Antifragilita, Democrazia

Ethics in Progress (ISSN 2084-9257). Vol. 8 (2017). No. 1, Art. \#8, pp. 117-146.

Creative Commons BY-SA 3.0

Doi: 10.14746/eip.2017.1.8 